Published in final edited form as:

Urol Oncol. 2020 January ; 38(1): 4.e1-4.e6. doi:10.1016/j.urolonc.2019.09.008.

\title{
Screening Logs From A Pilot Randomized Controlled Trial Of Radical Cystectomy Versus Chemoradiation Therapy For Muscle-Invasive Bladder Cancer
}

\author{
Dharam Kaushik, MD¹, Zheng Shi, MD², Michael A. Liss, MD¹, Hanzhang Wang, MS ${ }^{1}$, Richa \\ Priya Jha, MS ${ }^{1}$, Byeong Yeob Choi, PhD³ , Deepak K. Pruthi, MD', Chul Ha, MD², Ahmed M. \\ Mansour, MD ${ }^{1}$, Robert S. Svatek, MD ${ }^{1}$ \\ ${ }^{1}$ Department of Urology, University of Texas Health, San Antonio, TX \\ ${ }^{2}$ Department of Radiation Oncology, University of Texas Health, San Antonio, TX \\ ${ }^{3}$ Department of Epidemiology and Biostatistics, University of Texas Health, San Antonio, TX
}

\section{Abstract}

Introduction and Objectives: There is growing interest in a bladder preservation approach using chemoradiation therapy with transurethral resection of bladder tumor (TURBT), i.e., combined modality treatment (CMT), for muscle-invasive bladder cancer (MIBC). We have initiated a pilot study to determine feasibility of conducting a larger-scale clinical trial comparing CMT to radical cystectomy (RC) in patients with MIBC. Here we present the screening logs from the recruitment phase of this trial.

Methods: Patients who were diagnosed to have MIBC after TURBT between April 2016 and August 2017 and considered to be candidates for surgery were enrolled in this prospective, single center, randomized controlled pilot feasibility trial and scheduled to undergo RC (with neoadjuvant chemotherapy if appropriate) or CMT.

Results: Of 62 patients screened during the recruitment phase, only 5 were found to be suitable candidates for either treatment modality hence eligible for randomization. The reasons for exclusion were as follows: multifocal disease $(n=24,40 \%)$, variant histology $(n=15,25 \%)$, previous pelvic radiation $(\mathrm{n}=6,10 \%)$, severe lower urinary tract symptoms $(\mathrm{n}=5,8.3 \%)$, unwillingness to be enrolled $(n=8,13.3 \%)$ and receipt of neoadjuvant chemotherapy $(n=2,3.3 \%)$. One of the 5 eligible patients was randomized to CMT but was subsequently switched to RC because of a high tumor burden, one was randomized to RC, two were randomized to CMT but subsequently underwent TURBT and were considered ineligible because of extensive bladder disease, and one elected to undergo RC.

Corresponding author: Dharam Kaushik, MD, UT Health Science Center, Department of Urology, Division of Urologic Oncology, 7703 Floyd, Curl Drive, San Antonio, TX 78229-3900, USA, Tel: +1 210567 5676, Fax: +1 210567 6868, kaushik@uthscsa.edu.

Publisher's Disclaimer: This is a PDF file of an unedited manuscript that has been accepted for publication. As a service to our customers we are providing this early version of the manuscript. The manuscript will undergo copyediting, typesetting, and review of the resulting proof before it is published in its final citable form. Please note that during the production process errors may be discovered which could affect the content, and all legal disclaimers that apply to the journal pertain. 
Conclusions: We identified many patients with MIBC over a period of 16 months. However, the number of patients eligible to receive chemotherapy and in whom cystectomy and radiation therapy were both valid options was not as high as previously reported in retrospective CMT series. Many patients were excluded after TURBT. Our preliminary data indicate that only a very small subset of patients with MIBC are ideal candidates for CMT. Further research is required to identify patients who are suitable for CMT.

\section{Keywords}

Screening log; pilot study; radical cystectomy; chemoradiation therapy; overall survival

\section{Introduction}

In 2018, there were approximately 81,190 new bladder cancer cases and 17,240 bladder cancer-related deaths in the US alone. [1] Radical cystectomy (RC) is the gold standard treatment for muscle-invasive bladder cancer (MIBC). However, RC is associated with a 90day adverse event rate of $25 \%-65 \%$ and a perioperative mortality rate of $5 \%-10 \%$. [2, 3] Furthermore, patients who are treated surgically report poor overall satisfaction and healthrelated quality of life. [4] Given the high morbidity, mortality, and quality of life concerns with $\mathrm{RC}$, there is considerable interest in a bladder preservation approach. A combination of chemotherapy and radiotherapy with aggressive debulking transurethral resection of bladder tumor (TURBT), i.e., combined modality treatment (CMT), is now a widely used bladder preservation strategy. [5] CMT is a curative treatment option usually offered to patients who are not surgical candidates, i.e., those with extensive comorbidities and/or poor performance status. CMT as an acceptable modality for MIBC is now endorsed by multiple international cancer organizations that have developed evidence-based consensus guidelines, including the American Urologic Association, American Society of Clinical Oncology, Society of Urologic Oncology, National Comprehensive Cancer Network and European Association of Urology. [5, 6]

Retrospective studies have shown that CMT achieves a clinical response in the majority of patients and preserves the native bladder in more than $70 \%$ of cases while affording longterm survival rates comparable with those in contemporary cystectomy series. [7] Efstathiou et al reported respective 5-year disease-specific survival (DSS) and overall survival (OS) rates of $64 \%$ and 52\% at Massachusetts General Hospital. [7] Similarly, Mak et al found a 5year DSS rate of $71 \%$ and an OS rate of 57\% in their pooled analysis of the Radiation Therapy Oncology Group protocol data. [8] These rates are compatible with the 5-year DSS rate of $71.2 \%$ and OS rate of $58 \%$ reported to be achieved by contemporary RC. [9]

Our understanding of the role of CMT as an alternative to $\mathrm{RC}$ for MIBC is still evolving. Several comparative studies of RC versus CMT identified a reduction in the overall mortality risk after RC [10-13] while others found no difference in OS. [14-16] Most of the comparisons of RC with CMT have been either retrospective reviews of data in institutional cohort/national databases [10-16] or systematic reviews based on retrospective analyses and have had discordant findings. [17-19] Any direct comparison of CMT with RC must account for selection bias and confounding variables resulting from discordance between 
clinical and pathologic staging, functional status, and comorbidities. Given the paucity of data regarding the effects of these two treatment modalities on OS and lack of relevant RCTs, we performed this preliminary feasibility study with the aim of paving the way for a larger randomized clinical trial comparing the efficacy and safety of CMT and RC.

\section{Materials and Methods}

The protocol for this prospective, single-center, randomized non-inferiority pilot trial was approved by our institutional review board (IRB \#15-620H) and registered at ClinicalTrials.gov (). The primary outcome was the feasibility of randomizing patients to either radical cystectomy or combined modality therapy. Our main goal of this trial was to perform feasibility assessment and answering methodological questions, such as assessing recruitment difficulty in a surgical trial, coordination of the complex interactions between the various health care providers involved in treatment of bladder cancer patient.

A complete study schema is shown in Figure 1.

\section{Screening, consent and enrollment}

Potential study candidates were identified by research staff by review of our clinic schedules and by attending physicians, physician's assistants, and nurse practitioners in our urology clinics. The treating physician, physician's assistant, or nurse practitioner made the initial contact when a patient's pre-screening/chart review indicated that they may be eligible. Patients interested in participating in the trial could also contact the research staff for assessment of their eligibility to participate.

The technical aspects of the study were discussed with each potential participant by the principal investigator or another member of the research team. The following consent elements were discussed: the voluntary nature of participation, the purpose of the study, study procedures, risks and benefits, the collection of protected health information, confidentiality, and participant rights. Each patient was given a copy of the informed consent to read and consider and the opportunity to answer any questions. A copy of the signed consent form was provided to each study participant. A screening log of these patients was maintained.

The study inclusion criteria were as follows: a pathologic (histologic) diagnosis of primary carcinoma of the bladder (transitional cell cancer) within 8 weeks of registration; operable primary carcinoma of the bladder with histologic evidence of invasion of the muscularis propria and AJCC clinical stage T2-T4a, Nx or N0, M0; an adequately functioning bladder after careful evaluation by a urologist and as thorough a TURBT as was safely possible; and ability to tolerate systemic chemotherapy combined with pelvic radiation therapy or RC in the opinion of the treating physician. Patients were excluded if they were unable or unwilling to provide informed consent, had evidence of distant metastases or proven lymph node metastases, had previously received systemic chemotherapy (for any cancer) or pelvic radiation therapy, had clinical T4 bladder cancer, or had lymph node-positive bladder cancer with grossly enlarged pelvic or retroperitoneal lymph nodes. Radiation therapy protocol is 
uploaded as supplementary file \#2. Full list of inclusion and exclusion criteria are provided in the supplementary file $\# 2$.

\section{Randomization and statistical analysis}

Sample size calculation for pilot study: Assuming the $50 \%\left(\mathrm{p}_{2}\right)$ of patients randomized to radical cystectomy and $60 \%\left(\mathrm{p}_{1}\right)$ of patients randomized to chemoradiation will experience improvement in quality of life at 3 years relative to baseline and a $10 \%$ margin $(\Delta)$ of noninferiority, this study would require $\mathrm{n}=171$ subjects per group to attain $80 \%$ power for testing $\mathrm{H}_{\mathrm{o}}: \mathrm{p}_{1}-\mathrm{p}_{2} \leq \Delta$ versus $\mathrm{H}_{1}: \mathrm{p}_{1}-\mathrm{p}_{2}>\Delta$ with alpha $=5 \%$. We rounded $10 \%$ of that into $\mathrm{n}=30$ for a pilot study. [20]

Therefore, we planned to randomize 30 patients to receive either CMT $(n=15)$ or RC $( \pm$ neoadjuvant chemotherapy; $\mathrm{n}=15$ ). The study participants were assigned to the study groups by permuted block randomization with sequentially numbered, opaque sealed envelopes. We planned that all subjects would be randomized in a block size of 2 . A randomization list containing the sequence numbers and treatment assignments was prepared by a statistician and used as the sole basis for treatment assignment during the study. The association between categorical variables was evaluated using Fisher's exact test and the chi-square test. Differences in variables with a continuous distribution across dichotomous categories were assessed using the Mann-Whitney $U$ test. All statistical analyses were performed using Stata software (version 10.1; StataCorp LP, College Station, TX). All reported p-values were 2sided and statistical significance was set at $\mathrm{p}<0.05$.

\section{Results}

Sixty-two patients were screened for eligibility to participate in this trial between April 2016 and August 2017. The patient demographics and clinical staging information are shown in Table 1. Only 5 of the 62 patients were found to be ideal candidates for either treatment modality, hence, candidates for randomization. The reasons for exclusion were multifocal disease $(n=24,40 \%)$, variant histology $(n=15,25 \%)$, previous pelvic radiation $(n=6,10 \%)$, severe lower urinary tract symptoms $(\mathrm{n}=5,8.3 \%)$, and unwillingness to be enrolled in the study $(\mathrm{n}=8,13.3 \%$; Table 2$)$.

Of the 5 patients who consented to enrollment, one was randomized to CMT but was subsequently found to have a high tumor burden and switched to $\mathrm{RC}$, one was randomized to $\mathrm{RC}$, two patients were reassessed before randomization for TURBT and found to have extensive disease in the bladder that precluded CMT, and one opted for RC after enrollment (Figure 2).

\section{Discussion}

Our main objective of this study was assessing the feasibility of randomizing patients to either radical cystectomy or combined modality therapy. In addition, we evaluated the institutional capability to conduct the clinical trial by understanding specific ethical regulatory requirements, tools for randomization, obtaining informed consent from the study subjects and accruing patients for this study. In this single-institution pilot feasibility trial, 
we noticed that the proportion of patients with a diagnosis of MIBC in whom cystectomy and radiation therapy were both valid options was not as high as reported in the previous retrospective CMT series. [7, 16, 17, 21-23] This may reflect certain degree of selection bias. For example, Kulkarni et al [16] observed no difference between RC and CMT in regard to OS (35.7\% vs $39.3 \%$; $\mathrm{p}=0.63)$ in a single institutional series. They performed a robust statistical analysis to compare the two treatment modalities, however, their study was limited by selection bias in that patients with multifocal disease, a large tumor $(>5 \mathrm{~cm})$, hydronephrosis, or carcinoma in situ were not candidates for CMT. This pre-screening of patients in a retrospective study may have introduced a degree of selection bias that may have undermined the external validity of their results.

In our study, a considerable proportion of patients (40\%) were excluded after TURBT because of a high degree of multifocality and incomplete resection. Data from several studies have shown that patients treated by CMT without complete TURBT have a poor complete response rate and a higher likelihood of needing salvage RC. [24] Efstathiou et al reported salvage cystectomy rates of $22 \%$ in patients who had undergone complete TURBT and $42 \%$ in those with incomplete TURBT in their review of CMT data for 348 patients with clinical T2-T4a disease at Massachusetts General Hospital. [7] Furthermore, the patients who underwent complete TURBT had a higher complete remission rate than those who underwent incomplete TURBT (79\% vs 57\%; $\mathrm{p}<0.001$ ). Their updated analysis of 475 patients shows consistent results, i.e., patients requiring salvage cystectomy after failure of CMT had a significantly higher rate of initial incomplete TURBT ( $43 \%$ vs $24 \%$; $\mathrm{p}<0.001)$. Given that salvage cystectomy is associated with higher morbidity rates and poor cancerspecific survival, patient selection at the time of TURBT plays an essential role in the decision for CMT. [25] Approximately 13\% of our screening population had concomitant carcinoma in situ. CMT is not effective for carcinoma in situ because it is often diffuse and complete surgical removal is difficult, rendering this tumor non-responsive to a bladderpreserving strategy. [26]

Approximately $25 \%$ of our screened cohort had variant histology. This is an interesting observation given that squamous cell carcinoma, micropapillary carcinoma, and the nested and lympho-epithelioid variants are typically refractory to radiotherapy, usually present as locally advanced disease (cT3, cT4) with lymph node metastasis, and have a poor prognosis. [27-29]

Eight (13.3\%) of the 62 patients screened in this study expressed a strong preference for a particular treatment, thereby refusing enrollment. This reflects an inherent recruitment difficulty in a surgical trial, especially when the comparison arm is another treatment modality, such as radiotherapy. We attempted to mitigate some of the recruitment issues by simplifying the patient information sheet and consent process and by hiring a dedicated research coordinator (RJ), as well as ensuring that all study information was explained to the patients by either one of two urologic oncologists (DK, RSS) or a radiation oncologist (ZS). One of the main challenges faced by our group when recruiting patients for this trial was coordination of the complex interactions between the various health care providers involved in their treatment; for example, the referring physician (typically a community urologist who had counseled the patient to undergo RC), academic urologic oncology faculty, radiation and 
medical oncologists, residents and fellows in the urology and radiation oncology departments, and research nurses. After opening this clinical trial to recruitment, it took our group almost 6-8 months to streamline the logistics and flow of the trial. In an effort to improve patients' perception of this clinical trial and address issues of equipoise on the part of the urologists and radiation oncologists, all the clinicians involved in the trial regularly discussed potential patients and the possibility of enrollment at a biweekly genitourinary tumor board meeting. However, our screening log for this study reflects the challenges faced when undertaking complex surgical trials. Previous RCTs that have attempted to compare RC with CMT have suffered a similar fate. Notable among these was SPARE (Selective Bladder Preservation Against Radical Excision), which was a multicenter RCT comparing RC and CMT in patients with stage T2-3 N0 M0 MIBC who were suitable for either treatment strategy and had received three cycles of neoadjuvant chemotherapy.[30] Trial recruitment was challenging and below the predefined target, with only 45 patients recruited in 30 months ( $25 \mathrm{RC} ; 20 \mathrm{CMT}$ ). In that trial, fewer than 250 of more than 800 patients screened were potentially eligible for either treatment modality, which demonstrates the inherent difficulty in conducting such a trial. For a similar future clinical trial, we recommend maximizing use of social media and internet for spreading information regarding availability of clinical trial. This will be a cost-effective strategy and improve recruitment efficiency. In addition, utilization of print material such as posters, study brochure, paper flyers would enhance recruitment.

\section{Conclusions}

The screening log from the study reflects challenges faced in recruitment as well as accrual of patients for a randomized surgical trial. Only a very small subset of patients with MIBC are ideal candidates for CMT. Further research is required to identify patients who are suitable for this treatment strategy.

\section{Supplementary Material}

Refer to Web version on PubMed Central for supplementary material.

\section{Funding}

This project was supported by the National Center for Advancing Translational Sciences, National Institutes of Health (grant UL1 TR 001120 to DK). The content is solely the responsibility of the authors and does not necessarily represent the official views of the National Institutes of Health.

\section{References:}

[1]. Siegel RL, Miller KD, Jemal A. Cancer statistics, 2018. CA Cancer J Clin. 2018;68:7-30. [PubMed: 29313949]

[2]. Shabsigh A, Korets R, Vora KC, Brooks CM, Cronin AM, Savage C, et al. Defining early morbidity of radical cystectomy for patients with bladder cancer using a standardized reporting methodology. Eur Urol. 2009;55:164-74. [PubMed: 18675501]

[3]. Svatek RS, Fisher MB, Matin SF, Kamat AM, Grossman HB, Nogueras-Gonzalez GM, et al. Risk factor analysis in a contemporary cystectomy cohort using standardized reporting methodology and adverse event criteria. J Urol. 2010;183:929-34. [PubMed: 20083264] 
[4]. Yang LS, Shan BL, Shan LL, Chin P, Murray S, Ahmadi N, et al. A systematic review and metaanalysis of quality of life outcomes after radical cystectomy for bladder cancer. Surgical oncology. 2016;25:281-97. [PubMed: 27566035]

[5]. Gakis G, Efstathiou J, Lerner SP, Cookson MS, Keegan KA, Guru KA, et al. ICUD-EAU International Consultation on Bladder Cancer 2012: Radical cystectomy and bladder preservation for muscle-invasive urothelial carcinoma of the bladder. Eur Urol. 2013;63:45-57. [PubMed: 22917985]

[6]. Chang SS, Bochner BH, Chou R, Dreicer R, Kamat AM, Lerner SP, et al. Treatment of NonMetastatic Muscle-Invasive Bladder Cancer: AUA/ASCO/ASTRO/SUO Guideline. J Urol. 2017;198:552-9. [PubMed: 28456635]

[7]. Efstathiou JA, Spiegel DY, Shipley WU, Heney NM, Kaufman DS, Niemierko A, et al. Long-term outcomes of selective bladder preservation by combined-modality therapy for invasive bladder cancer: the MGH experience. Eur Urol. 2012;61:705-11. [PubMed: 22101114]

[8]. Mak RH, Hunt D, Shipley WU, Efstathiou JA, Tester WJ, Hagan MP, et al. Long-term outcomes in patients with muscle-invasive bladder cancer after selective bladder-preserving combinedmodality therapy: a pooled analysis of Radiation Therapy Oncology Group protocols 8802, 8903, 9506, 9706, 9906, and 0233. J Clin Oncol. 2014;32:3801-9. [PubMed: 25366678]

[9]. Hautmann RE, de Petriconi RC, Pfeiffer C, Volkmer BG. Radical cystectomy for urothelial carcinoma of the bladder without neoadjuvant or adjuvant therapy: long-term results in 1100 patients. Eur Urol. 2012;61:1039-47. [PubMed: 22381169]

[10]. Ritch CR, Balise R, Soodana Prakash N, Alonzo D, Almengo K, Alameddine M, et al. Propensity matched comparative analysis of survival following chemoradiation or radical cystectomy for muscle invasive bladder cancer. BJU Int. 2017.

[11]. Seisen T, Sun M, Lipsitz SR, Abdollah F, Leow JJ, Menon M, et al. Comparative Effectiveness of Trimodal Therapy Versus Radical Cystectomy for Localized Muscle-invasive Urothelial Carcinoma of the Bladder. Eur Urol. 2017;72:483-7. [PubMed: 28412065]

[12]. Cahn DB, Handorf EA, Ghiraldi EM, Ristau BT, Geynisman DM, Churilla TM, et al. Contemporary use trends and survival outcomes in patients undergoing radical cystectomy or bladder-preservation therapy for muscle-invasive bladder cancer. Cancer. 2017;123:4337-45. [PubMed: 28743162]

[13]. Kaushik D, Wang H, Michalek J, Liss MA, Liu Q, Jha RP, et al. Chemoradiation Versus Radical Cystectomy for Muscle-Invasive Bladder Cancer: A Propensity Score-Weighted Comparative Analysis Using the National Cancer Database. Urology. 2019.

[14]. Gofrit ON, Nof R, Meirovitz A, Pode D, Frank S, Katz R, et al. Radical cystectomy vs. chemoradiation in T2-4aN0M0 bladder cancer: a case-control study. Urol Oncol. 2015;33:19.e1-.e5.

[15]. Kim YJ, Byun SJ, Ahn H, Kim CS, Hong BS, Yoo S, et al. Comparison of outcomes between trimodal therapy and radical cystectomy in muscle-invasive bladder cancer: a propensity score matching analysis. Oncotarget. 2017;8:68996-9004. [PubMed: 28978174]

[16]. Kulkarni GS, Hermanns T, Wei Y, Bhindi B, Satkunasivam R, Athanasopoulos P, et al. Propensity Score Analysis of Radical Cystectomy Versus Bladder-Sparing Trimodal Therapy in the Setting of a Multidisciplinary Bladder Cancer Clinic. J Clin Oncol. 2017;35:2299-305. [PubMed: 28410011]

[17]. Arcangeli G, Strigari L, Arcangeli S. Radical cystectomy versus organ-sparing trimodality treatment in muscle-invasive bladder cancer: A systematic review of clinical trials. Critical reviews in oncology/hematology. 2015;95:387-96. [PubMed: 25934521]

[18]. Ploussard G, Daneshmand S, Efstathiou JA, Herr HW, James ND, Rodel CM, et al. Critical analysis of bladder sparing with trimodal therapy in muscle-invasive bladder cancer: a systematic review. Eur Urol. 2014;66:120-37. [PubMed: 24613684]

[19]. Vashistha V, Wang H, Mazzone A, Liss MA, Svatek RS, Schleicher M, et al. Radical Cystectomy Compared to Combined Modality Treatment for Muscle-Invasive Bladder Cancer: A Systematic Review and Meta-Analysis. Int J Radiat Oncol Biol Phys. 2017;97:1002-20. [PubMed: 28332983] 
[20]. Stallard N. Optimal sample sizes for phase II clinical trials and pilot studies. Stat Med. 2012;31:1031-42. [PubMed: 22052407]

[21]. AlGizawy SM EH, Abdel-Wanis ME, Abdel Rhaeem AM. Trimodality bladder-sparing approach versus radical cystectomy for invasive bladder cancer. Journal of Radiotherapy in Practice. 2007:428-37.

[22]. Bekelman JE, Handorf EA, Guzzo T, Evan Pollack C, Christodouleas J, Resnick MJ, et al. Radical cystectomy versus bladder-preserving therapy for muscle-invasive urothelial carcinoma: examining confounding and misclassification biasin cancer observational comparative effectiveness research. Value Health. 2013;16:610-8. [PubMed: 23796296]

[23]. Gofrit ON, Nof R, Meirovitz A, Pode D, Frank S, Katz R, et al. Radical cystectomy vs. chemoradiation in T2-4aN0M0 bladder cancer: a case-control study. Urol Oncol. 2015;33:19.e15.

[24]. Giacalone NJ, Shipley WU, Clayman RH, Niemierko A, Drumm M, Heney NM, et al. Long-term Outcomes After Bladder-preserving Tri-modality Therapy for Patients with Muscle-invasive Bladder Cancer: An Updated Analysis of the Massachusetts General Hospital Experience. Eur Urol. 2017;71:952-60. [PubMed: 28081860]

[25]. Calderone CE, Lerner SP, Taylor JM. The case for salvage cystectomy after pelvic radiation. Minerva urologica e nefrologica $=$ The Italian journal of urology and nephrology. 2016;68:16171. [PubMed: 26982375]

[26]. Premo C, Apolo AB, Agarwal PK, Citrin DE. Trimodality therapy in bladder cancer: who, what, and when? The Urologic clinics of North America. 2015;42:169-80, vii. [PubMed: 25882559]

[27]. Chalasani V, Chin JL, Izawa JI. Histologic variants of urothelial bladder cancer and nonurothelial histology in bladder cancer. Canadian Urological Association journal = Journal de l'Association des urologues du Canada. 2009;3:S193-8.

[28]. Kassouf W, Spiess PE, Siefker-Radtke A, Swanson D, Grossman HB, Kamat AM, et al. Outcome and patterns of recurrence of nonbilharzial pure squamous cell carcinoma of the bladder: a contemporary review of The University of Texas M D Anderson Cancer Center experience. Cancer. 2007;110:764-9. [PubMed: 17614317]

[29]. Rogers CG, Palapattu GS, Shariat SF, Karakiewicz PI, Bastian PJ, Lotan Y, et al. Clinical outcomes following radical cystectomy for primary nontransitional cell carcinoma of the bladder compared to transitional cell carcinoma of the bladder. J Urol. 2006;175:2048-53; discussion 53. [PubMed: 16697800]

[30]. Huddart RA, Birtle A, Maynard L, Beresford M, Blazeby J, Donovan J, et al. Clinical and patient-reported outcomes of SPARE - a randomised feasibility study of selective bladder preservation versus radical cystectomy. BJU Int. 2017;120:639-50. [PubMed: 28453896] 
- In patients with muscle-invasive bladder cancer, number of patients in whom radical cystectomy and chemoradiation therapy (CMT) are both valid options is not as high as previously reported in retrospective series

- Many patients were excluded from CMT treatment after transurethral resection of bladder tumor

- Only a very small subset of patients with muscle-invasive bladder cancer are ideal candidates for CMT 


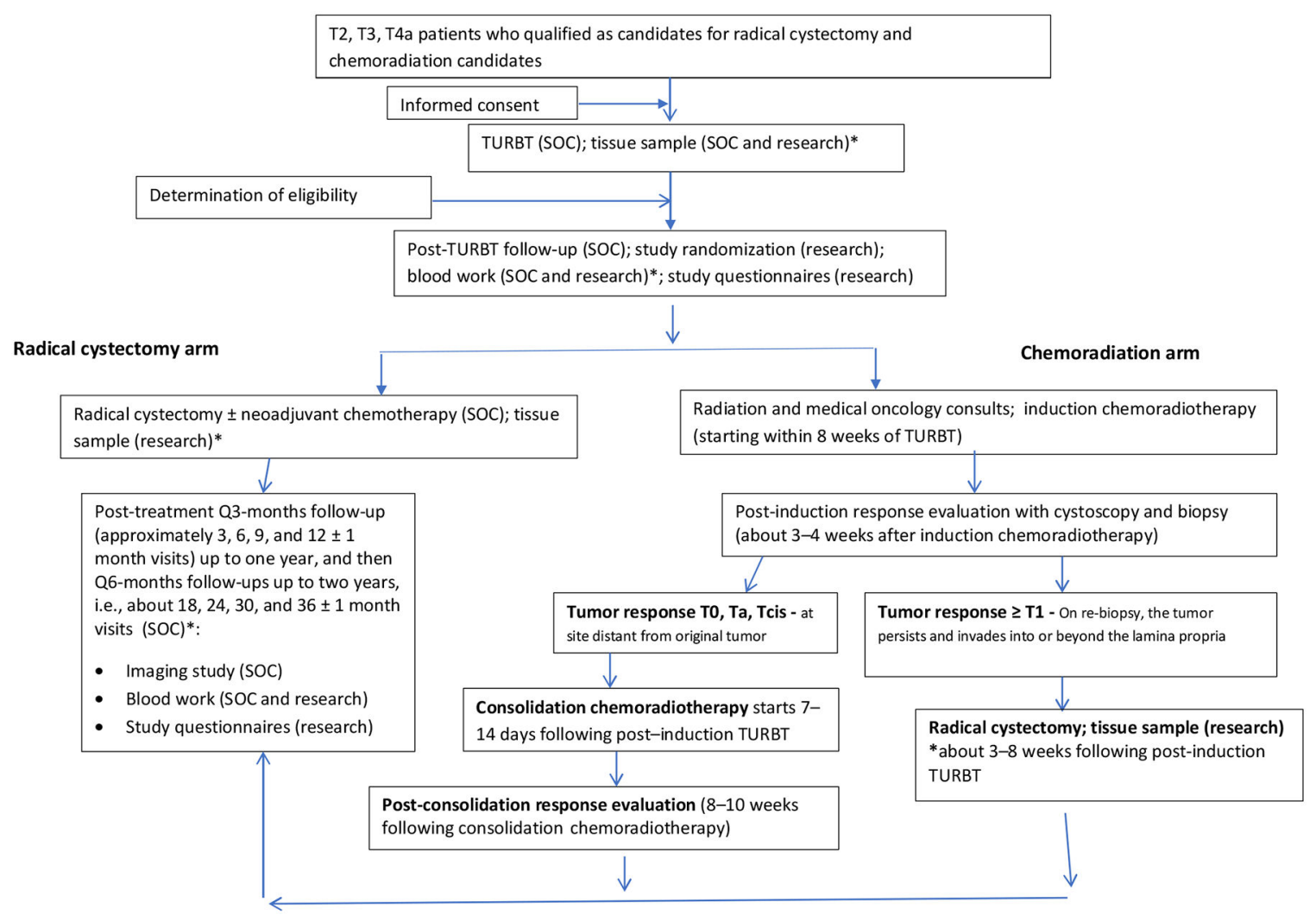

Figure 1.

Study synopsis: Radical cystectomy vs chemoradiation study

Abbreviations: SOC, standard of care; TURBT, transurethral resection of bladder tumor 


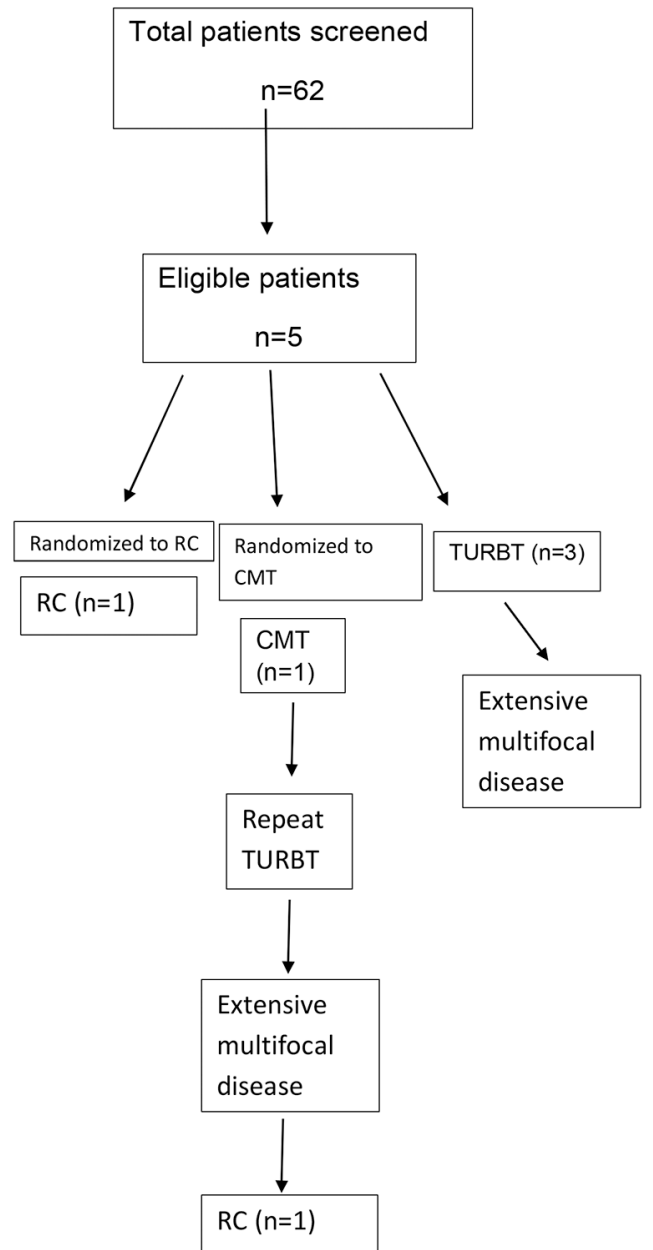

Figure 2.

CONSORT flow diagram demonstrating the study cohort.

Abbreviations: CMT, combined modality treatment; RC, radical cystectomy; TURBT, transurethral resection of bladder tumor 


\section{Table 1}

Demographics and clinical staging of study participants

\begin{tabular}{ll}
\hline Age, median (IQR) & $71(62-78)$ \\
\hline Sex, n (\%) & \\
\hline Male & $52(83.87)$ \\
\hline Female & $10(16.13)$ \\
\hline BMI, median (IQR) & $26.65(23.6-30.42)$ \\
\hline Clinical stage, n (\%) & \\
\hline cT2 & $45(72.58)$ \\
\hline cT3 & $9(14.52)$ \\
\hline cT4 & $8(12.90)$ \\
\hline Ethnicity, n (\%) & \\
\hline Hispanic & $20(32.26)$ \\
\hline Non-Hispanic & $41(66.13)$ \\
\hline Other & $1(1.61)$ \\
\hline
\end{tabular}

Abbreviations: BMI, body mass index; IQR, interquartile range 
Table 2:

Reasons for exclusion from participation in the study

\begin{tabular}{|c|c|}
\hline Reason for exclusion & $\%(n=60)$ \\
\hline 1. Multifocal disease & $40 \%(n=24)$ \\
\hline A. Multifocal tumor with concurrent severe CIS & $13.3 \%(8)$ \\
\hline B. Upper tract involvement & $8.3 \%(5)$ \\
\hline C. Prostatic stromal involvement & $8.3 \%(5)$ \\
\hline D. Vaginal involvement & $5 \%(3)$ \\
\hline E. Pelvic lymphadenopathy & $5 \%(3)$ \\
\hline 2. Variant histology & $25 \%(n=15)$ \\
\hline A. Squamous cell carcinoma & $11.6 \%(7)$ \\
\hline B. Micropapillary carcinoma & $3.3 \%(2)$ \\
\hline C. Small cell carcinoma & $3.3 \%(2)$ \\
\hline D. Other (nested variant, lymphoepitheloid) & $6.6 \%(4)$ \\
\hline 3. Previous pelvic radiation & $10 \%(\mathrm{n}=6)$ \\
\hline 4. Severe lower urinary tract symptoms (urethral stricture, multiple sclerosis) & $8.3 \%(\mathrm{n}=5)$ \\
\hline 5. Patient refused enrollment & $13.3 \%(\mathrm{n}=8)$ \\
\hline 6. Already received neoadjuvant chemotherapy & $3.3 \%(n=2)$ \\
\hline
\end{tabular}

